\title{
Immunohistochemistry for the detection of BRCA1 and $B R C A 2$ proteins in patients with ovarian cancer: a systematic review
}

\author{
Lorena Alves Teixeira ำ , Francisco Jose Candido dos Reis
}

\begin{abstract}
- Additional material is published online only. To view please visit the journal online (http://dx.doi.org/10.1136/ jclinpath-2019-206276).

Postgraduate Program in Gynecology and Obstetrics, Department of Gynecology and Obstetrics, Ribeirao Preto Medical School, University of Sao Paulo, Ribeirao Preto, Sao Paulo, Brazil
\end{abstract}

\section{Correspondence to}

Dr Francisco Jose Candido dos Reis, Department of Gynecology and Obstetrics, Ribeirao Preto Medical School, University of Sao Paulo, Ribeirao Preto, Brazil; fjcreis@usp.br

Received 15 October 2019 Accepted 28 October 2019 Published Online First 12 November 2019

\begin{abstract}
Background Loss of function in either breast cancer type 1 susceptibility protein (BRCA1) or breast cancer type 2 susceptibility protein (BRCA2) is a major risk factor for epithelial ovarian cancer (EOC) development. BRCA1 or BRCA2 deficiencies are associated with short-term prognosis and might have importance for the treatment of women with the disease. However, the screening of all possible mechanisms of dysfunction is expensive, time-consuming and difficult to apply in clinical practice. On the other hand, immunohistochemistry (IHC) is a simple and reliable method to access the expression of several proteins in tumour tissues.
\end{abstract}

Materials and methods This systematic review aims to evaluate the current usage of IHC to detect BRCA1 and BRCA2 deficiencies in EOC. We searched and evaluated all primary literature on the use of $\mathrm{IHC}$ for evaluating BRCA1 and BRCA2 proteins expression in EOC. The main concepts for the search were: ovarian neoplasms, IHC, BRCA1 and BRCA2.

Results Forty-four studies from 925 unique titles were included. A total of 4206 tumour samples were evaluated for BRCA1 and 1041 for BRCA2 expression. Twelve BRCA1 primary antibodies were used in 41 studies, and the most common was the MS110 clone (75.6\%). Seven BRCA2 primary antibodies were used in ten studies. Using the cut-off of $10 \%, 47.0 \%$ of EOCs are associated with loss of BRCA1 and $34.5 \%$ with the loss of BRCA2 expression.

Conclusion IHC was effective to detect loss of BRCA1 protein expression in EOC; however, data on BRCA2 expression were heterogeneous and difficult to interpret.

\section{INTRODUCTION}

Epithelial ovarian cancer (EOC) comprises a group of heterogeneous diseases with distinct morphology, etiopathogenesis, molecular features, biologic behaviour and clinical outcomes. ${ }^{1-3}$ Based on histopathology, immunohistochemistry (IHC) and molecular analysis, malignant epithelial ovarian tumours are classified in five main subtypes: highgrade serous carcinoma (HGSC), low-grade serous, mucinous, endometrioid and clear cell carcinomas. ${ }^{4}$ HGSC, the most common subtype, is frequently associated with BRCA1 and BRCA2 abnormalities. ${ }^{14}$

$B R C A 1$ and BRCA2 genes encode proteins involved in repairing damaged DNA. ${ }^{5}$ Homologous recombination-mediated double-strand breaks repair deficiency occurs in approximately half of HGSC cases. ${ }^{1}$ BRCA1 or BRCA2 dysfunction can result from pathogenic germline variants, pathogenic somatic variants or epigenetic silencing. ${ }^{16}$ There are several methods for the detection of BRCA1 and BRCA2 dysfunction. IHC is the mainstay of laboratory techniques for diagnosis, localisation and detection of dysfunctional proteins. ${ }^{7}$ IHC is an inexpensive and accessible method. Several studies have examined BRCA1 and BRCA2 using this immunostaining. Some antibodies can identify the majority of alterations that result in protein truncation. ${ }^{8}$ However, protocols, assessment and interpretation of results vary extensively. ${ }^{10-14}$

We aimed to review all published evidence on IHC usage for the detection of BRCA1 and BRCA2 proteins in patients with EOC to identify the current protocols and to inform future studies.

\section{METHODS}

This study followed the quality reporting guidelines set by the Preferred Reporting Items for Systematic Reviews and Meta-Analyses (PRISMA) statement. ${ }^{15}$

The inclusion criteria were: (1) observational studies or clinical trials involving women with EOC, including primary ovarian, fallopian tube or peritoneal carcinomas; (2) analysis of BRCA1 or BRCA2 expression by IHC and (3) adequate description of IHC method. The exclusion criteria were: (1) studies on cell culture or animals; (2) case reports; (3) non-original articles; (4) articles with overlapped subjects; (5) duplicate publication or (6) studies without full-text available.

\section{Information sources and literature search strategy}

Two independent reviewers (LAT and FJCdR) performed a systematic and comprehensive electronic literature search to identify the potentially eligible studies. We searched on Excerpta Medica database (EMBASE), Medical Literature Analysis and Retrieval System Online (MEDLINE) via PubMed, Web of Science, Scopus databases and grey literature up to July 2019 without language or period restrictions. The search included a combination of free text keywords, Emtree terms and Medical Subject Heading terms, modified according to each database, as well as synonyms and truncated terms (see online supplementary file 1 S1-S4 for complete search strategy). Finally, we also manually checked the reference lists of all included articles to find additional studies. 


\section{Study selection}

We used the Zotero software to screen for duplicates and Rayyan platform ${ }^{16}$ to perform the titles and abstracts screening. In the case of duplicated studies or different studies that included the same group of patients, we selected the most informative, complete or with more recent data. Discordancies between the reviewers were resolved by consensus.

\section{Data extraction and synthesis of results}

We used four standardised data extraction forms (article information, study characteristics, protein expression by IHC methods and results) designed into the Research Electronic Data Capture $^{17}$ online platform hosted at University of Sao Paulo. Authors were contacted by email to provide missing data or fulltext when it was not available. The studies that failed to provide essential elements or available full-text were excluded. Extracted data were carefully reviewed. Results were presented as proportions and summary tables.

\section{Assessment of study quality}

Due to the exploratory nature of this systematic review, we included all studies meeting the eligible criteria, irrespective of individual study quality.

\section{RESULTS}

\section{Study selection}

The primary search identified 1550 studies. The screening of the reference lists resulted in four additional articles and the search in grey literature in two additional studies. After removing the duplicates, 925 titles and abstracts were thoroughly screened, resulting in 110 articles selected for full-text review. Among the 110 manuscripts selected for full-text analysis, 16 studies were excluded due to the following reasons: no available fulltext $(n=2)$, abstract or oral presentation $(n=13)$ and duplicate study $(n=1)$. Ninety-four full-text manuscripts were assessed for eligibility, among them, 50 studies were excluded due to the following reasons: not about ovarian cancer $(n=5)$, non-invasive ovarian tumours $(n=1)$, non-original article $(n=1)$, same subjects included in more than one study $(n=6)$, non-human studies $(n=5)$, insufficient information $(n=7)$, unanswered email $(n=1)$, case report $(n=3)$ and no IHC for BRCA1 or BRCA2 $(n=21)$.

A total of 44 studies were included in this systematic review. ${ }^{1012} 1318-58$ The flow chart outlining the study design is shown in figure 1 . The details of the excluded full-text articles and the respective reasons are given in online supplementary file $1 \mathrm{~S} 5$.

\section{Characteristics of eligible studies}

The main characteristics of the 44 included studies are summarised in online supplementary file 1 S6 . Overall, 1 study

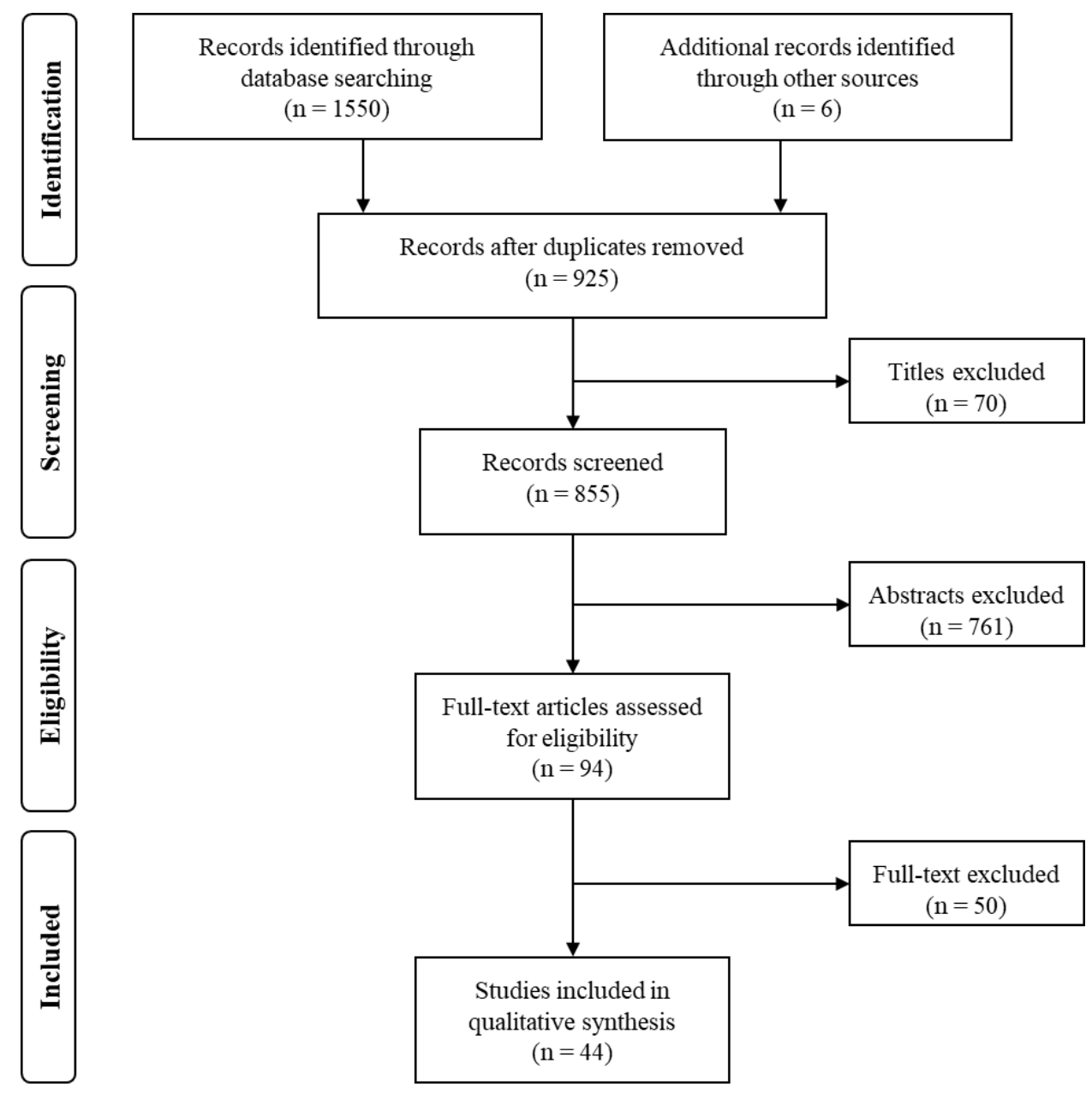

Figure 1 Flow diagram according to the Preferred Reporting Items for Systematic Reviews and Meta-Analyses guidelines for the literature search and identification of studies using immunohistochemistry to access BRCA1 or BRCA2 expression. 
was in Bulgarian, ${ }^{35} 1$ study was in Chinese, ${ }^{51} 1$ study was in Spanish, ${ }^{22} 1$ study was in Turkish ${ }^{25}$ and all the other 40 studies were in English. A total of 4735 patients with EOC, diagnosed from 1985 to 2015, were included. Thirty-four studies assessed BRCA1, three studies BRCA2 and seven studies both proteins. There were data on BRCA1 for 4312 cases, on BRCA2 for 1041 cases and on both proteins for 617 cases. The median sample size for study was 87, and the mean 108 cases (range 4-393). All studies used the International Federation of Gynecology and Obstetrics (FIGO) staging system, except one that used TNM staging. ${ }^{34}$ The details of the IHC methods used in the 44 studies are shown in online supplementary file 1 S7.

\section{Immunohistochemistry for BRCA1}

According to the main purposes of the BRCA1 IHC expression studies, $20(48.8 \%)$ aimed to verify EOC prognosis (2954 tumours), 9 (21.9\%) clinicopathological characteristics (584 tumours), 4 (9.7\%) gene promoter methylation (321 tumours), $2(4.9 \%)$ aimed to screen for germline mutation (73 tumours) and $6(14.6 \%)$ studies to detect BRCA1 deficiency associated with multiple biological mechanisms (347 tumours).

For BRCA1 IHC technique (see online supplementary file 1 S7), formalin-fixed paraffin-embedded (FFPE) tissue was used in 40 studies and frozen tissue in one study. Whole-slide (WS) tissue sections were used in 25 studies, tissue microarray (TMA) in 13 studies, in 2 studies the type of section was not described and in 1 study the IHC was performed pooling WS and TMA. The thickness of the sections was described in 30 studies, which varied from 3 to $5 \mu \mathrm{m}$, with 15 (30\%) using $4 \mu \mathrm{m}$. For the remaining 11 studies, the thickness of the sections was not reported.

Twelve BRCA1 primary antibodies were used, and in one study, the primary antibody was not described. The anti-BRCA1 mouse monoclonal clone MS110 was used in 31 (75.6\%) studies. One study used two antibodies ${ }^{29}$ and one study used six antibodies. Slides were incubated with each diluted primary antibody ranging from 1:10 to $1: 5000$. In 12 studies the dilutions were not described. The incubated period ranged from $30 \mathrm{~min}$ to 16 hours with the temperature ranging from $4^{\circ} \mathrm{C}$ to $37^{\circ} \mathrm{C}$ (see online supplementary file $1 \mathrm{~S} 7$ ).

We identified three systems to score BRCA1 expression. In 18 studies, scoring was done by the percentage of positive tumour cells independently of the intensity, in 2 studies, scoring was done only by the intensity of positive tumour cells and in 19 studies the scoring was done considering the percentage of positive tumour cells and the intensity of the staining. One study did not describe the scoring system used. In 28 studies only nuclear staining was scored, in 10 studies nuclear and cytoplasmic expression were scored, in 1 study only cytoplasmic expression was scored and 2 studies did not report this information. The blinded assessment was carried out in 25 of $40(62.5 \%)$ studies, and control samples were described in 34 of $40(85.0 \%)$ studies.

The cut-off value to consider loss of BRCA1 expression was $10 \%$ of positive cells in 25 studies, $5 \%$ in 5 studies, $1 \%$ in 2 studies, in 8 studies only the complete absence of positive tumour cells was considered loss of expression and 2 studies used other cut-off scores. The proportion of loss of BRCA1 expression according to the cut-off value is shown in table 1 . The pooled proportion of loss of BRCA1 protein expression was $47.7 \%$ with cut-off $10 \%, 72.7 \%$ with cut-off $5 \%, 45.5 \%$ with cut-off $1 \%$ and $51.3 \%$ with no positive tumour cell.

\section{Immunohistochemistry for BRCA2}

According to the main purposes of the BRCA2 IHC expression studies, seven $(70.0 \%)$ studies aimed to verify prognosis (805 tumours), one (10.0\%) aimed to study clinicopathological features (161 tumours), and two (20.0\%) studies aimed to detect BRCA2 deficiency from multiple biological mechanisms (58 tumours).

For BRCA2 technique (see online supplementary file 1 S7), FFPE tissue blocks were used in all ten studies. WS tissue sections were used in one study, TMAs in seven, and in two studies, the types of tissue sections were not described. The thickness of the sections was described in five (50\%) studies and varied from $4 \mu \mathrm{m}$ (one study) to $5 \mu \mathrm{m}$ (four studies). Four studies $(40 \%)$ used the anti-BRCA2 mouse monoclonal clone Mab2476,

\begin{tabular}{|c|c|c|c|c|c|c|}
\hline Cut-off & Histology & Tumour type & Studies (n) & Total (n) & Negative BRCA1 (n) & Negative BRCA1 (\%) \\
\hline \multirow[t]{7}{*}{$10 \%$} & Mixed & Sporadic & 8 & 1282 & 649 & 50.62 \\
\hline & Mixed & Sporadic and hereditary or familial & 3 & 72 & 39 & 54.17 \\
\hline & Mixed & Unknown & 7 & 588 & 296 & 50.34 \\
\hline & Serous & Unknown & 4 & 383 & 142 & 37.08 \\
\hline & HGSC & Sporadic and hereditary or familial & 2 & 291 & 110 & 37.80 \\
\hline & PPSC & Sporadic and hereditary or familial & 1 & 14 & 2 & 14.28 \\
\hline & Sub-total & & 25 & 2630 & 1238 & 47.07 \\
\hline \multirow[t]{4}{*}{$5 \%$} & HGSC & Sporadic and hereditary or familial & 1 & 17 & 1 & 5.88 \\
\hline & HGSC & Unknown & 2 & 327 & 252 & 77.06 \\
\hline & Endometrioid & Unknown & 1 & 45 & 30 & 66.67 \\
\hline & Sub-total & & 4 & 389 & 283 & 72.75 \\
\hline $1 \%$ & Mixed & Sporadic and hereditary or familial & 2 & 180 & 82 & 45.55 \\
\hline \multirow[t]{4}{*}{ Any } & Mixed & Sporadic & 3 & 431 & 207 & 48.03 \\
\hline & Mixed & Sporadic and hereditary or familial & 2 & 193 & 71 & 26.78 \\
\hline & Mixed & Unknown & 3 & 348 & 221 & 63.50 \\
\hline & Sub-total & & 8 & 972 & 499 & 51.34 \\
\hline H-score $\geq 47$ & Mixed & Unknown & 1 & 28 & 23 & 82.14 \\
\hline H-score $>70$ & HGSC & Sporadic & 1 & 139 & 117 & 84.2 \\
\hline
\end{tabular}

HGSC, High-grade serous carcinoma; PPSC, Primary peritoneal serous cancer. 


\begin{tabular}{|c|c|c|c|c|c|c|}
\hline Cut-off & Histology & Tumour type & Studies (n) & Total (n) & Negative BRCA2 (n) & Negative BRCA2 (\%) \\
\hline \multirow[t]{4}{*}{$10 \%$} & Mixed & Sporadic & 1 & 115 & 49 & 42.50 \\
\hline & Mixed & Unknown & 2 & 215 & 115 & 53.50 \\
\hline & HGSC & Sporadic & 1 & 156 & 53 & 34.00 \\
\hline & PPSC & Sporadic and hereditary or familial & 1 & 14 & 0 & 00.00 \\
\hline $5 \%$ & Mixed & Unknown & 1 & 274 & 218 & 79.50 \\
\hline$\geq 2$ score & Mixed & Unknown & 1 & 209 & 176 & 84.00 \\
\hline$\geq 4$ score & HGSC & Unknown & 1 & 150 & 123 & 82.00 \\
\hline
\end{tabular}

HGSC, High-grade serous carcinoma; PPSC, Primary peritoneal serous cancer.

and six other antibodies were utilised for the remaining studies. Antibody dilution was described in six studies and varied from $1: 30$ to $1: 300$ and the incubation time varied from $60 \mathrm{~min}$ to 16 hours, at a temperature from $4^{\circ} \mathrm{C}$ to $37^{\circ} \mathrm{C}$.

There were two systems to score BRCA2 expression. In five studies scoring was done by the percentage of positive tumour cells independently of the intensity, and in five studies the scoring was done considering the percentage of positive tumour cells and the intensity of the staining. In eight studies only nuclear expression was considered, and two studies did not report the BRCA2 localisation. The cut-off for considering loss of BRCA2 expression was $10 \%$ in five studies, and 5\% in two studies. On the remaining two studies, one used the cut-off $\geq 2$, and other one used $\geq 4$.

The blinded analyses were carried out for 8 of $10(80.0 \%)$ studies stated, although in one more study automated analyses were used. The control samples were used in nine of ten studies; only one did not report this information.

The proportion of loss of BRCA2 protein expression according to the cut-off value is shown in table 2 . The pooled proportion of loss of BRCA2 expression was $42.3 \%$ with cut-off $10 \%$, $79.5 \%$ with cut-off $5 \%$.

\section{DISCUSSION}

We reviewed all published studies on IHC for BRCA1 and BRCA2 in EOC. BRCA1 studies were more frequent, and the methods presented some homogeneity. The results when using the anti-BRCA1 mouse monoclonal clone MS110 antibody and the cut-off of $10 \%$ of positive tumour cells are compatible with the expected proportion of BRCA1 deficient ovarian tumours. On the other hand, BRCA2 studies were less frequent, the methods were heterogeneous and no protocol identified BRCA2 deficient in an expected proportion tumour.

The main strength of this article is the comprehensivity of the search. We evaluated virtually all available literature about IHC for BRCA1 and BRCA2 in ovarian cancer. On the other hand, our conclusions might be limited by the heterogeneity of the methods published. Most of the included articles are retrospective and therefore susceptible to selection bias. Also, we were not able to access the real accuracy IHC because the published studies lack a gold standard for BRCA1 or BRCA2 deficiency.

Loss of function, reduced expression or mutational inactivation of $B R C A 1$ or $B R C A 2$ were reported to increase sensitivity to platinum agents. ${ }^{54}$ The defective BRCA1 or BRCA2 recognised in ovarian cancer is medically and therapeutic relevant, but the analysis of genetic and genomics is complex, expensive, impractical as a screening method for detection in all patients and it does not include the evaluation of BRCA1 promoter methylation or mRNA expression levels. ${ }^{159}$ The IHC method is inexpensive and less labor-intensive than DNA analysis. ${ }^{7}$

The negative expression of BRCA1 or BRCA2 can be a marker of homologous recombination deficiency. Germline or somatic pathogenic BRCA1 and BRCA2 variants, or BRCA1 promoter methylation are potential mechanisms of homologous recombination deficiency that can be present in up to $50 \%$ of cases of malignant epithelial ovarian tumours. ${ }^{60}$ The described frequency of negative or low expression of BRCA1 ranged from $5.88 \%$ to $84.2 \%$. The heterogeneity of methods used may be responsible for this variation. When $10 \%$ of positive cells was used as the cut-off, around $47 \%$ of cases were considered BRCA1 deficient.

There are several ways to score IHC gene expression and to choose cut-off levels. For example, a breast tumour with nuclear staining of oestrogen receptor $\geq 1 \%$ is considered positive because it has been proved sensitive to tamoxifen. ${ }^{6162}$ Based on current data, $10 \%$ of positive cells would be a recommended cutoff for BRCA1 deficiency. However, one interesting approach would be to evaluate the cut-off associated with the response to drugs targeting homologous recombination deficiency such as polyADP ribose polymerase (PARP) inhibitors. ${ }^{63}$

Studies on IHC for the detection of BRCA2 deficiency were very heterogeneous in methods and results. The pooled frequency of BRCA2 deficient was much higher than the expected frequency. The frequency of BRCA2 germline or somatic mutation in ovarian cancer is less than $10 \% .{ }^{6465}$ These results suggest that the current IHC protocols for BRCA2 lack sensitivity to detect the abnormal protein.

The most consistent results on IHC for BRCA1 in ovarian cancer were obtained with the anti-BRCA1 mouse monoclonal clone MS110 antibody and a cut-off of $10 \%$ of positive cells. We recommend further studies on cut-off determination based on tumour sensitivity to drugs like PARP inhibitors. On IHC for BRCA2, further studies are needed to develop antibodies with better sensitivity to detect the normal protein in ovarian tumours.

\section{TAKE HOME MESSAGES}

- Anti-BRCA1 mouse monoclonal clone MS110 antibody is the most effective antibody to detect BRCA1 deficienciescurrently available.

- The cut-off of $10 \%$ of positive tumour cells seems to be adequate to identify BRCA1 proficient EOCs.

- New antibodies are needed to access BRCA2 in EOC.

Handling editor Runjan Chetty.

Contributors Both authors contributed enough to this article to be considered as authors. 
Funding LAT (grant number 130162/2017-5) was funded by the National Council for Scientific and Technological Development (CNPq). FJCdR (grant number 303210/2018-4) was funded by the CNPq. This study was financed in part by the Coordenação de Aperfeiçoamento de Pessoal de Nível Superior, Brasil-Finance Code 001.

Competing interests None declared.

Patient consent for publication Not required.

Provenance and peer review Not commissioned; internally peer reviewed.

ORCID iDs

Lorena Alves Teixeira http://orcid.org/0000-0003-1053-2046

Francisco Jose Candido dos Reis http://orcid.org/0000-0001-5758-5917

\section{REFERENCES}

1 Bell D, Berchuck A, Birrer M, et al. Integrated genomic analyses of ovarian carcinoma. Nature 2011;474:609-15.

2 Kurman RJ, Shih I-M. The dualistic model of ovarian carcinogenesis: revisited, revised, and expanded. Am J Pathol 2016;186:733-47.

3 Meinhold-Heerlein I, Hauptmann S. The heterogeneity of ovarian cancer. Arch Gynecol Obstet 2014;289:237-9.

4 Prat J, D’Angelo E, Espinosa I. Ovarian carcinomas: at least five different diseases with distinct histological features and molecular genetics. Hum Pathol 2018;80:11-27.

5 Scully R. Interactions between BRCA proteins and DNA structure. Exp Cell Res 2001;264:67-73.

6 Konstantinopoulos PA, Ceccaldi R, Shapiro Gl, et al. Homologous recombination deficiency: exploiting the fundamental vulnerability of ovarian cancer. Cancer Discov 2015;5:1137-54.

7 Kuhn E, Ayhan A. Diagnostic immunohistochemistry in gynaecological neoplasia: a brief survey of the most common scenarios. J Clin Pathol 2018;71:98-109.

8 Ellison $\mathrm{G}$, Huang $\mathrm{S}$, Carr $\mathrm{H}$, et al. A reliable method for the detection of BRCA1 and BRCA2 mutations in fixed tumour tissue utilising multiplex PCR-based targeted next generation sequencing. BMC Clin Pathol 2015;15.

9 Plaskocinska I, Shipman H, Drummond J, et al. New paradigms for BRCA1/BRCA2 testing in women with ovarian cancer: results of the genetic testing in epithelial ovarian cancer (GTEOC) study. J Med Genet 2016;53:655-61.

10 Carser JE, Quinn JE, Michie CO, et al. BRCA1 is both a prognostic and predictive biomarker of response to chemotherapy in sporadic epithelial ovarian cancer. Gynecol Oncol 2011;123:492-8

11 Garg K, Levine DA, Olvera N, et al. BRCA1 immunohistochemistry in a molecularly characterized cohort of ovarian high-grade serous carcinomas. Am J Surg Pathol 2013:37:138-46.

12 Meisel JL, Hyman DM, Garg K, et al. The performance of BRCA1 immunohistochemistry for detecting germline, somatic, and epigenetic BRCA1 loss in high-grade serous ovarian cancer. Ann Oncol 2014;25:2372-8.

13 Sun C-Y, Su T-F, Li N, et al. A chemotherapy response classifier based on support vector machines for high-grade serous ovarian carcinoma. Oncotarget 2016;7:3245-54.

14 Perez-Valles A. The usefulness of antibodies to the BRCA1 protein in detecting the mutated BRCA1 gene. An immunohistochemical study. J Clin Pathol 2001;54:476-80.

15 Liberati A, Altman DG, Tetzlaff J, et al. The PRISMA statement for reporting systematic reviews and meta-analyses of studies that evaluate healthcare interventions: explanation and elaboration. BMJ 2009;339:b2700.

16 Ouzzani M, Hammady H, Fedorowicz Z, et al. Rayyan-a web and mobile APP for systematic reviews. Syst Rev 2016;5:210.

17 Harris PA, Taylor R, Thielke R, et al. Research electronic data capture (REDCap)--a metadata-driven methodology and workflow process for providing translational research informatics support. J Biomed Inform 2009:42:377-81.

18 Al-Kashwan TA, Al-Bedairi FJ, Al-Midhaffer RH. Significant role of loss or reduced BRCA1 gene expression in clinical implication of ovarian cancer. Kufa Med J 2017:17:62-76.

19 Bai X, Fu Y, Xue H, et al. BRCA1 promoter hypermethylation in sporadic epithelial ovarian carcinoma: Association with low expression of $B R C A 1$, improved survival and co-expression of DNA methyltransferases. Oncol Lett 2014;7:1088-96.

20 Baldwin RL, Nemeth E, Tran H, et al. Brca1 promoter region hypermethylation in ovarian carcinoma: a population-based study. Cancer Res 2000;60:5329-33.

21 Cho DH, Park HS, Park S-H, et al. The expression of DBC1/CCAR2 is associated with poor prognosis of ovarian carcinoma. J Ovarian Res 2015;8.

22 Fernández A, Mora E, Villegas J, et al. Expresión de BRCA1 en mujeres Con neoplasias epiteliales de ovario del Estado Carabobo, Venezuela. Rev Venez Oncol 2017:29:112-22

23 Furukawa N, Ohno S, Kasai T, et al. Evaluation of the BRCA1/2 mutation as a prognostic marker in primary peritoneal serous cancer. J Obstet Gynecol 2013:03:653-7.

24 Gan A, Green AR, Nolan CC, et al. Poly(adenosine diphosphate-ribose) polymerase expression in BRCA-proficient ovarian high-grade serous carcinoma; association with patient survival. Hum Pathol 2013;44:1638-47.
25 Guenes N, Yilmaz F, Uzunlar AK. Grading of the ovarian serous cystadencarcinomas, and its correlation with BRCA1 and p53 immunoreactivity. Turk Klin TIP Bilim Derg 2007;27:335-43

26 Hjortkjær M, Waldstrøm M, Jakobsen A, et al. The prognostic value of BRCA1 and PARP expression in epithelial ovarian carcinoma: immunohistochemical detection. Int J Gynecol Pathol 2017:36:180-9.

27 Ju L-L, Zhao CY, Ye K-F, et al. Expression and clinical implication of Beclin1, HMGB1, p62, survivin, BRCA1 and ERCC1 in epithelial ovarian tumor tissues. Eur Rev Med Pharmacol Sci 2016;20:1993-2003.

28 Kaern J, Aghmesheh M, Nesland JM, et al. Prognostic factors in ovarian carcinoma stage III patients. Can biomarkers improve the prediction of short- and long-term survivors? Int I Gynecol Cancer 2005;15:1014-22.

29 Kashima K, Oite T, Aoki Y, et al. Screening of BRCA1 mutation using immunohistochemical staining with C-Terminal and $\mathrm{N}$-Terminal antibodies in familial ovarian cancers. Jpn J Cancer Res 2000:91:399-409.

30 Lesnock JL, Darcy KM, Tian C, et al. BRCA1 expression and improved survival in ovarian cancer patients treated with intraperitoneal cisplatin and paclitaxel: a gynecologic Oncology Group study. Br J Cancer 2013;108:1231-7.

31 Li M, Li H, Liu F, et al. Characterization of ovarian clear cell carcinoma using target drug-based molecular biomarkers: implications for personalized cancer therapy. J Ovarian Res 2017:10.

32 McAlpine JN, Porter $\mathrm{H}$, Köbel $\mathrm{M}$, et al. BRCA1 and BRCA2 mutations correlate with TP53 abnormalities and presence of immune cell infiltrates in ovarian high-grade serous carcinoma. Mod Pathol 2012:25:740-50.

33 McMillen BD, Aponte MM, Liu Z, et al. Expression analysis of MIR182 and its associated target genes in advanced ovarian carcinoma. Mod Pathol 2012;25:1644-53.

34 Nomura H, Kataoka F, Aoki D, et al. Expression of potential biomarkers associated with homologous recombination repair in patients with ovarian or triple-negative breast cancer. CBM 2016;16:145-52.

35 Popovska S, Ivanov I, Dineva T, et al. Morphologically qnd immunohistochemically based screening criteria for selection of patients with possible mutation of BRCA gene in primary ovarian cancer. Akush Ginekol 2014;53:21-8.

36 Pradjatmo H, Dasuki D, Anwar M, et al. Methylation status and immunohistochemistry of BRCA1 in epithelial ovarian cancer. Asian Pac J Cancer Prev 2014:15:9479-85.

37 Press JZ, De Luca A, Boyd N, et al. Ovarian carcinomas with genetic and epigenetic BRCA1 loss have distinct molecular abnormalities. BMC Cancer 2008:8.

38 Radosa MP, Häfner N, Camara O, et al. Loss of BRCA1 protein expression as indicator of the BRCAness phenotype is associated with favorable overall survival after complete resection of sporadic ovarian cancer. Int I Gynecol Cancer 2011:21:1399-406.

39 Russell PA, Pharoah PDP, De Foy K, et al. Frequent loss of BRCA1 mRNA and protein expression in sporadic ovarian cancers. Int. J. Cancer 2000;87:317-21.

40 Shawky AE-A, El-Hafez AA, El-Tantawy D, et al. No association between BRCA immunohistochemical expression and tumor grade, stage or overall survival in platinum-treated epithelial ovarian cancer patients. Asian Pac J Cancer Prev 2014; 15:4275-9.

41 Shilpa V, Bhagat R, Premalata CS, et al. BRCA1 promoter hypermethylation and protein expression in ovarian carcinoma — an Indian study. Tumor Biol 2014:35:4277-84.

42 Sirisabya N, Manchana T, Termrungreunglert W, et al. Prevalence of BRCA1 expression in epithelial ovarian cancer: immunohistochemical study. J Med Assoc Thai 2007:90:9-14

43 Skytte A-B, Waldstrom M, Rasmussen AA, et al. Identification of BRCA1-deficient ovarian cancers. Acta Obstet Gynecol Scand 2011;90:593-9.

44 Strickland KC, Howitt BE, Shukla SA, et al. Association and prognostic significance of BRCA1/2-mutation status with neoantigen load, number of tumor-infiltrating lymphocytes and expression of PD-1/PD-L1 in high grade serous ovarian cancer. Oncotarget 2016:7:13587-98.

45 Swisher EM, Gonzalez RM, Taniguchi T, et al. Methylation and protein expression of DNA repair genes: association with chemotherapy exposure and survival in sporadic ovarian and peritoneal carcinomas. Mol Cancer 2009;8:48

46 Thrall $\mathrm{M}$, Gallion HH, Kryscio R, et al. Brca1 expression in a large series of sporadic ovarian carcinomas: a gynecologic Oncology Group study. Int J Gynecol Cancer 2006;16 Suppl 1:166-71

47 Vaz FH, Machado PM, Brandão RD, et al. Familial breast/ovarian cancer and BRCA1/2 genetic screening: the role of immunohistochemistry as an additional method in the selection of patients. J Histochem Cytochem 2007:55:1105-13.

48 Vorrius TR, Snyder K, Pica-Mendez A, et al. Immunohistochemical detection of BRCA-1 and BRCA-2 expression in human breast and ovarian tumors. J Histotechnol 2009;32:202-3.

49 Wang C, Horiuchi A, Imai T, et al. Expression of BRCA1 protein in benign, borderline, and malignant epithelial ovarian neoplasms and its relationship to methylation and allelic loss of the BRCA1 gene. J Pathol 2004;202:215-23.

50 Wang Z, Liu Y, Lu L, et al. Fibrillin-1, induced by Aurora-A but inhibited by BRCA2, promotes ovarian cancer metastasis. Oncotarget 2015;6:6670-83.

51 Wang L, Dong Y, Li Q, et al. [Clinicopathologic features observation of ovarian transitional cell tumors]. Zhonghua Bing Li Xue Za Zhi 2015;44:118-22. 
52 Weberpals JI, Tu D, Squire JA, et al. Breast cancer 1 (BRCA1) protein expression as a prognostic marker in sporadic epithelial ovarian carcinoma: an NCIC CTG OV.16 correlative study. Annals of Oncology 2011;22:2403-10.

53 Yang G, Chang B, Yang F, et al. Aurora kinase A promotes ovarian tumorigenesis through dysregulation of the cell cycle and suppression of BRCA2. Clin Cancer Res 2010;16:3171-81.

54 Yang F, Guo X, Yang G, et al. AURKA and BRCA2 expression highly correlate with prognosis of endometrioid ovarian carcinoma. Mod Pathol 2011;24:836-45.

55 Ye Q, Chen L, Yin X, et al. Development of serous ovarian cancer is associated with the expression of homologous recombination pathway proteins. Pathol Oncol Res 2014;20:931-8

56 Zhang M, Zhuang G, Sun X, et al. Risk prediction model for epithelial ovarian cancer using molecular markers and clinical characteristics. J Ovarian Res 2015;8:67.

57 Zheng W, Luo F, Lu JJ, et al. Reduction of BRCA1 expression in sporadic ovarian cancer. Gynecol Oncol 2000;76:294-300.

58 Sallum LF, Andrade L, Bastos Eloy da Costa L, et al. Brca1, Ki67, and $\beta$-catenin immunoexpression is not related to differentiation, platinum response, or prognosis in women with low- and high-grade serous ovarian carcinoma. Int I Gynecol Cancer 2018;28:437-47.
59 Lord CJ, Ashworth A. BRCAness revisited. Nat Rev Cancer 2016;16:110-20.

60 Moschetta M, George A, Kaye SB, et al. BRCA somatic mutations and epigenetic BRCA modifications in serous ovarian cancer. Ann Oncol 2016:27:1449-55.

61 Hicks D, Dell'Orto P, Falzon M, et al. Immunohistochemical performance of estrogen and progesterone receptor antibodies on the Dako Omnis staining platform: evaluation in multicenter studies. Appl Immunohistochem Mol Morphol 2017:25:313-9.

62 Hammond MEH, Hayes DF, Dowsett M, et al. American Society of clinical Oncology/College of American pathologists guideline recommendations for immunohistochemical testing of estrogen and progesterone receptors in breast cancer. JCO 2010;28:2784-95.

63 Moore K, Colombo N, Scambia G, et al. Maintenance olaparib in patients with newly diagnosed advanced ovarian cancer. N Engl J Med 2018;379:2495-505.

64 Elvin JA, He Y, Sun J, et al. Comprehensive genomic profiling (CGP) with loss of heterozygosity $(\mathrm{LOH})$ to identify therapeutically relevant subsets of ovarian cancer (OC). JCO 2017;35:5512.

65 Norquist BM, Brady MF, Harrell MI, et al. Mutations in homologous recombination genes and outcomes in ovarian carcinoma patients in GOG 218: an NRG Oncology/ Gynecologic Oncology Group study. Clin Cancer Res 2018;24:777-83. 